\title{
Investigation into use of double-layer grid structures as frequency selective surfaces for buildings'
}

\author{
P. Callaghan*, R. Huelin, Md Hasanuzzaman Sagor \\ Faculty of Engineering and Science, University of Greenwich, Chatham Maritime, Kent ME4 \\ 4TB, UK \\ *P.Callaghan@greenwich.ac.uk
}

\begin{abstract}
A simple Double-Layer Grid plane wave filter structure is proposed that can provide multiple transmission bands for cellular phone frequencies but with a reflection band for WLAN signals. The approach offers ease of construction making it applicable to building applications. A parametric study using simulation supported by simple experimental data investigates the proposed, novel design.
\end{abstract}

\section{Introduction}

The increase of wireless systems over the last couple of decades has resulted in overcrowding in the electromagnetic spectrum. System designers have developed novel modulation techniques and multiantenna solutions, such as MIMO, to increase data capacity over a given wireless channel. Perhaps the greatest challenge is presented by the modern office building, wherein there is a high density of users. Propagation through the structure of the building structure itself has to be mitigated and some interesting solutions are presented in [1] particularly with regard to steel reinforced materials. However, a major challenge in the design of the building is to permit 'macro-cell' communications (i.e. mobile phone) to propagate through the whole structure, but to limit propagation of internal wireless LAN to a specific area, such as a single office, allowing re-use of the WLAN band in adjacent rooms [2]. To this end much research has been carried out into the application of Frequency Selective Surface (FSS) technology to the design of the modern building - the aim being to restrict wireless propagation to within a 'room', by making the walls act as a plane wave filter [3].

Research into FSS design for buildings [3] has focused principally on realizing novel element structures to meet the wide frequency bandwidths involved - control is needed from $400 \mathrm{MHz}$ (for TETRA mobile radio) up to $2.4 \mathrm{GHz}$ (WLAN frequencies), demanding a 6:1 frequency response. The upper 'useful' frequency of an FSS is usually limited by the appearance of 'trapped waves' [4] as the periodicity approaches a wavelength. Most researchers have limited themselves to investigating novel single layer FSS structures [5]. Some work has been undertaken into double-layer structures - although to improve the band shape provided by a single-layer resonance. In [3] two cascaded FSS were demonstrated to widen the bandwidth of the resonance whilst some workers have used two different patterns in close proximity to generate FSS with a wide band spacing [6]. Fabrication has also been a significant challenge and some recent work has proposed solutions for integrating FSS onto glass [7] (for windows and

This paper is a postprint of a paper submitted to and accepted for publication in [journal] and is subject to Institution of Engineering and Technology Copyright. The copy of record is available at IET Digital Library 
partitions) and even blinds [8]. The double-layer design on glass in [7] is of interest as it offers a doubleresonance response, similar to the work in this paper, but using a different approach. Naturally propagation within a building is also dependent on scattering and an insight into this has been given in [9] which investigated a wall made using 'Smart bricks' employing FSS.

Fabrication of FSS is a significant challenge, and many of the structures proposed would be difficult to build on a large scale - certainly to cover walls, floors and ceilings. Rather this paper re-visits the problem of allowing transmission of waves through steel reinforced material in buildings and look to see if can take advantage of the steel reinforcement, rather than consider it a drawback, as in [2]. It is well known that cascading 2 or more gratings can generate transmission bands [10]. We review this approach and investigate two embedded metallic meshes, as illustrated in Fig. 1, and tune their plane wave transmission line effect to create passbands. Note one or more of these grids could be a metallic reinforcing mesh (no evaluation of the mechanical properties has been made at this point - the focus was achieving desirable plane wave properties. For this work we took a design goal of passing cellular radio signals ( $900 \mathrm{MHz}$ and $1800 \mathrm{MHz}$ bands) but with an option to reflect WLAN (2.4 GHz) signals.

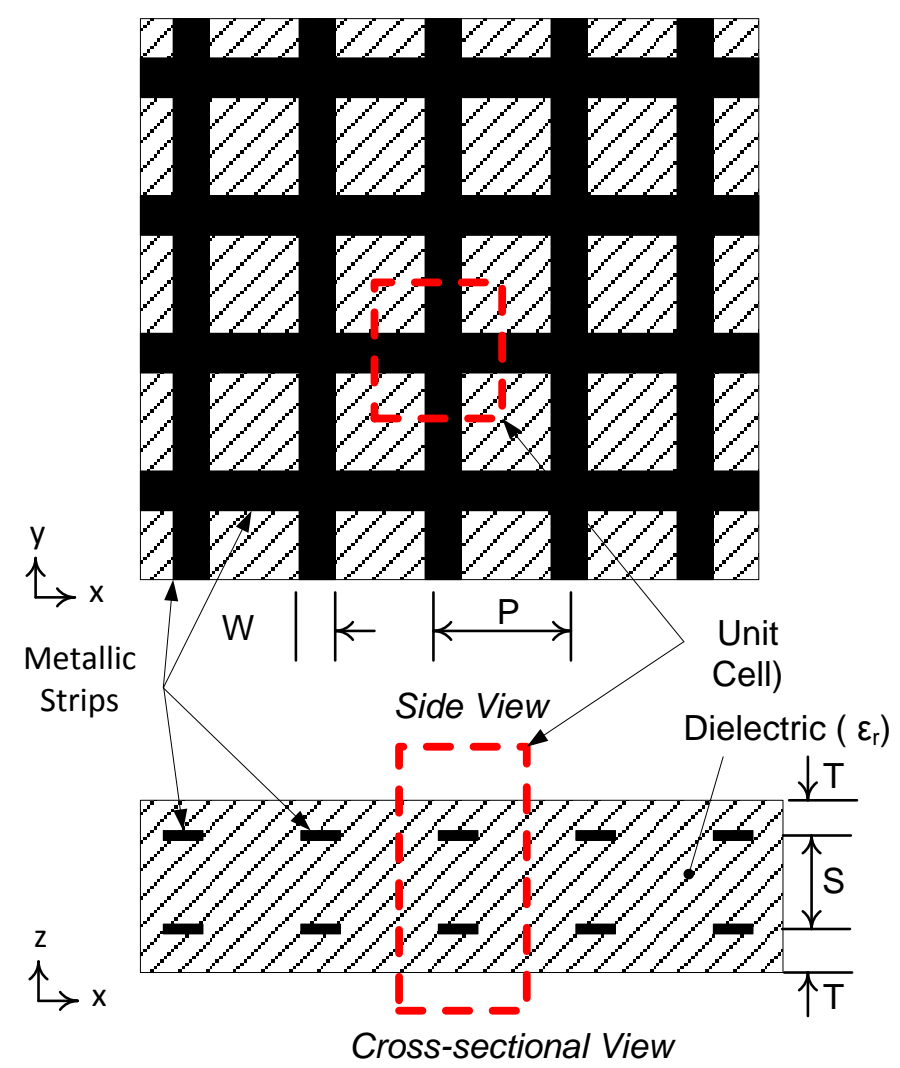

Fig. 1: Outline of proposed Double-Layer Grid Filter

This paper is a postprint of a paper submitted to and accepted for publication in [journal] and is subject to Institution of Engineering and Technology Copyright. The copy of record is available at IET Digital Library 


\section{Double Layer Grid Filter Design}

The Double-Layer Grid Filter structure is presented in Fig.1. It consists of 2 strip gratings, or grids, separated in space by a dielectric former. It is well known that a strip grating can be modelled as an inductance in an equivalent circuit representation of a plane wave structure [11]. Here the electric field of the incident wave must be parallel to the wires; hence they reflect the wave, with an 'inductive' effect. At low frequencies, defined by the spacing between the wires being much smaller than the wavelength, the grating starts to act like a reflector and no signal passes through - hence providing 'screening' at lower frequencies blocking wireless signals, as discussed in [2].

Note in this paper grids are considered in the theoretical study. A grid provides an inductive effect to both horizontal and vertical polarization, so would be applicable to the general building problem. For experimental work a strip grating was used which demonstrates the concept practically although for a single polarization. Similarly we assume infinitely thin, flat conductors - but finite thickness, or even round wires will give similar results.

Using the transmission line analogy, as illustrated in Fig.2, it is easily understood that a second grating placed approximately one quarter of a wavelength away causes a resonance between the two layers. The inductance of the second grating is transformed to be capacitive at the plane of the first grating, creating a parallel resonant circuit having a transmission band. The frequency of the resonance is determined by the inductance of each grid and the spacing between them, so a transmission line circuit simulator was used initially to calculate suitable values. It was also expected that a second passband would be formed, likely at 3 times the frequency of the first resonance (i.e. when the spacing was three-quarters of a wavelength). However, the transmission line simulator calculated that the frequency of the second resonance could be much lower, nearer only twice that of the first resonance. For example, the frequency response of a circuit with two $2 \mathrm{nH}$ inductors separated by a transmission line of impedance $50 \mathrm{Ohm}$, and length $130 \mathrm{~mm}$ is shown in Fig. 3. This provides transmission bands around $1 \mathrm{GHz}$ and at $2 \mathrm{GHz}$, to suit the cellular mobile phone bands. This structure is equivalent to placing two grids $130 \mathrm{~mm}$ apart in free space. Also plotted in Fig. 3 is the transmission response with a single inductor of value $1 \mathrm{nH}$ (i.e. the $2 \mathrm{x}$ $2 \mathrm{nH}$ in parallel) - illustrating a transmission loss of around $14 \mathrm{~dB}$ at $1 \mathrm{GHz}$, and much higher at lower frequencies. Clearly using two grids rather than one can resolve 'screening' problems, for specific bands.

This paper is a postprint of a paper submitted to and accepted for publication in [journal] and is subject to Institution of Engineering and Technology Copyright. The copy of record is available at IET Digital Library 


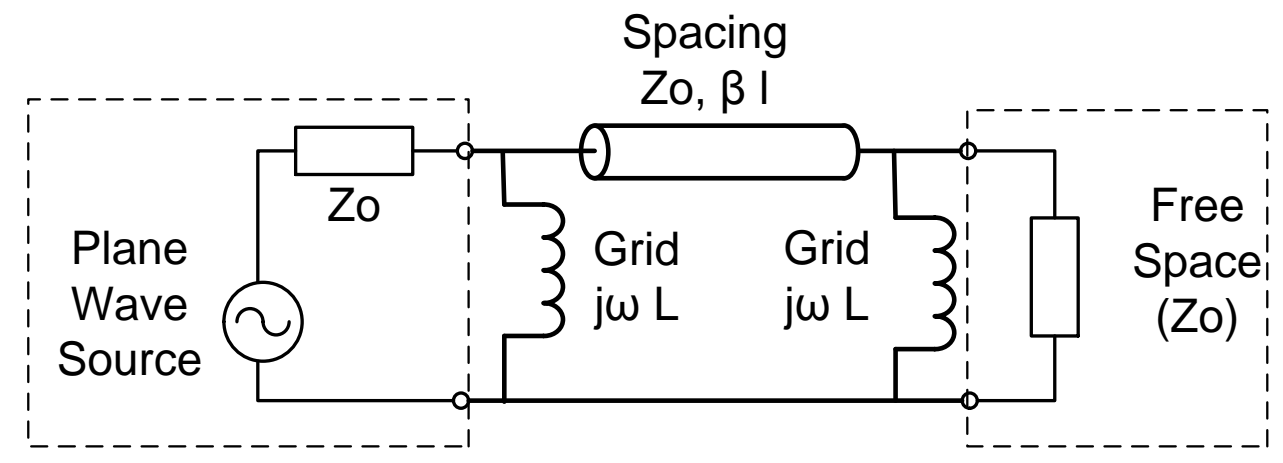

Fig. 2: Equivalent circuit representation of Double-Layer Grid Filter

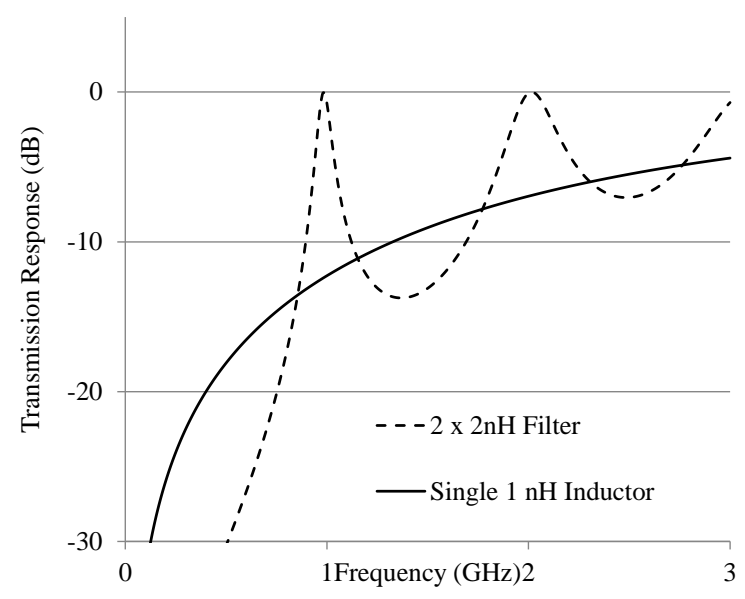

Fig. 3: Frequency Response of Transmission Line Filter Circuit

Whilst a 'passband' has been achieved at $1 \mathrm{GHz}$ in Fig.3 the bandwidth is quite narrow. Currently the UHF mobile phone bands are being extended significantly (i.e. for LTE) such that this tuned passband may have limited applicability. However, the band width can be altered by adjusting the parameters of the transmission line circuit. To investigate this 4 structures were tuned to $\sim 900 \mathrm{MHz}$ using different values of inductance and transmission line length, as give in Table 1. The frequency responses of these four different designs are compared in Fig 4.

Table 1 Details of Transmission Line Filter in Fig. 4

\begin{tabular}{ccc}
\hline $\begin{array}{c}\text { Inductor } \\
\text { Value }(\mathrm{nH})\end{array}$ & $\begin{array}{c}\text { Transmission Line } \\
\text { Length }(\mathrm{mm})\end{array}$ & $\begin{array}{c}\text { Bandwidth at } \\
800 \mathrm{MHz}\end{array}$ \\
\hline & & \\
1 & 175 & $1 \%$ \\
5 & 160 & $5 \%$ \\
10 & 130 & $30 \%$ \\
\hline
\end{tabular}

This paper is a postprint of a paper submitted to and accepted for publication in [journal] and is subject to Institution of Engineering and Technology Copyright. The copy of record is available at IET Digital Library 
It is seen that by increasing the inductance the bandwidth increases. Indeed for $10 \mathrm{nH}$ the bandwidth is so wide that the bands begin to merge, and form a low pass structure. Rather than a single metallic reinforcement causing 'screening' and loss of signal, a double layer structure could provide multiple resonances allowing good transmission of mobile signals.

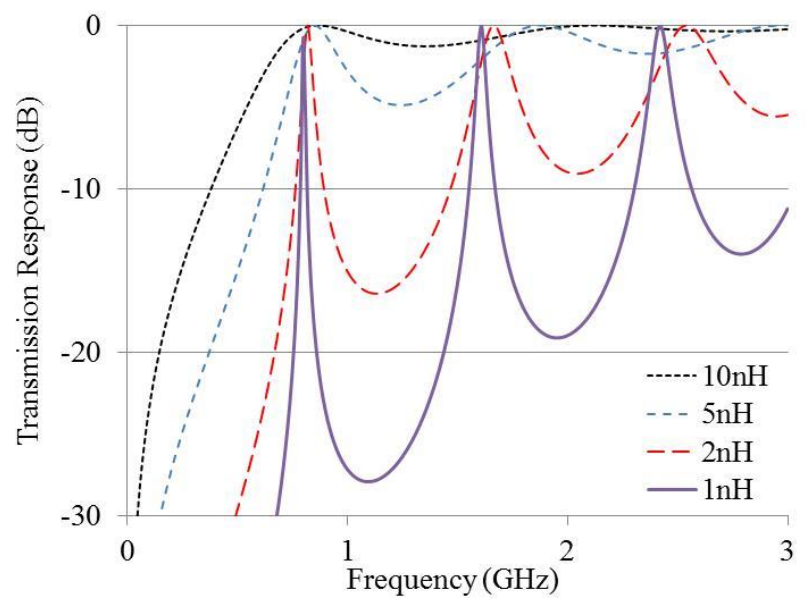

Fig.4: Transmission Line Filters using different Inductances

\section{Plane Wave Structure}

The responses shown in Figs. 3 and 4 assume that each grid behaves like an inductor - but in practice there are significant differences. At lower frequencies the variation of the grid reactance using closed form expressions [12] has been shown to be similar to an inductance provided that the ratio of $\mathrm{W}$ to $\mathrm{P}$ as defined in Fig. 1 is much less than 1 . The reactance is also very dependent upon incidence angle - this is explored further in section 5. However, as the wavelength becomes smaller than the periodicity of the structure the equivalent circuit analogy breaks down. Above this point diffraction effects occur (generation of additional waves). Hence in designing a plane wave filter using two grids the periodicity of the grids ( $\mathrm{P}$ in Fig. 1) is a critical design parameter.

An additional design goal was to achieve a reflection band at $2.4 \mathrm{GHz}$, typically needed to create isolated 'WLAN zones'. In Fig. 4 stopbands can be provided using small values of 'inductance' - but at the loss of the desired wider passbands. However it is well known that when the wavelength becomes equal to the periodicity a surface wave can be excited that results in a deep, narrow reflection band [4]. Here we propose to make the periodicity a wavelength at $2.4 \mathrm{GHz}$ (i.e. $\lambda=125 \mathrm{~mm}$ ) to deliberately generate a surface wave resonance to provide the desired reflection band. A grid structure having $\mathrm{W}=20 \mathrm{~mm}$ and $\mathrm{P}=125 \mathrm{~mm}$ was calculated to give $\sim 5 \mathrm{nH}$ inductance (using [12]) and then the double layer structure, with

This paper is a postprint of a paper submitted to and accepted for publication in [journal] and is subject to Institution of Engineering and Technology Copyright. The copy of record is available at IET Digital Library 
spacing of $130 \mathrm{~mm}$ was simulated using CST Microwave Studio. CST MWS has a Floquet mode model for infinite periodic array - this includes diffraction waves so can model a grating accurately. The array is modelled as a unit cell, as indicated in Fig. 1, with a 'Floquet Port' on one side of the structure with the wave propagating in the $\mathrm{z}$ direction. There are two fundamental propagating modes one having an electric field in the y-direction and the other in the x-direction (i.e. orthogonal). As the incidence angle is varied one of these modes has a Transverse Magnetic (TM) polarisation whilst the other is Transverse Electric (TE). As will be seen the plane of polarisation can have a significant impact. The simulations from CST for normal incidence are compared to the equivalent circuit model response in Fig. 5.

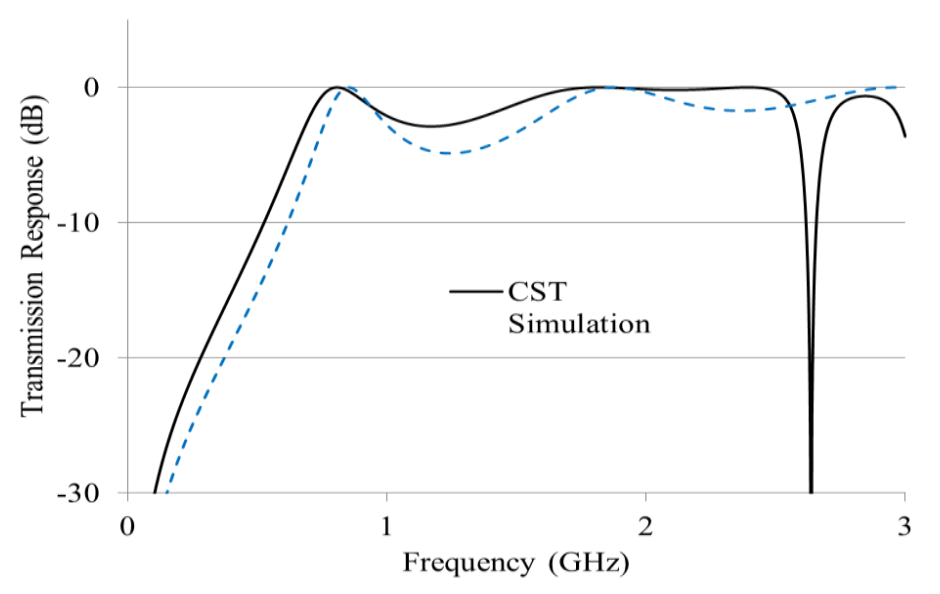

Fig.5: Comparison of simple Lumped Element Circuit Model to Full-Wave EM simulation for Double Layer Grid Filter

As can be seen from Fig. 5 there is reasonable agreement between the equivalent circuit model and a practical plane wave structure. - both giving the two passbands around 900 and $1800 \mathrm{MHz}$. However, there is a significant difference with the appearance of a surface wave null at higher frequencies. This is higher than expected, at $2.7 \mathrm{GHz}$, but clearly the structure offers a very desirable transmission / reflection characteristic.

\section{Effect of Dielectric}

The response in Fig. 5 is for 'free-standing' gratings. In practice the gratings need to be supported by a dielectric material. CST was used again to investigate the response, at normal incidence, for a double grating structure immersed in various dielectrics. To assess the practical application of this work the permittivities to be used need to be representative of typical building materials. For this, measured values for permittivity were adopted from [13] where sample building materials have been characterized. Here [13] report a variation of permittivity and loss tangent with frequency but for this study the 'average' value was used, with the dielectrics assumed to be non-dispersive. Also the dimensions of the grid and spacing, for a permittivity of 1.0, were optimized using CST to give passbands at $900 \mathrm{MHz}$ and $1800 \mathrm{MHz}$ with the

This paper is a postprint of a paper submitted to and accepted for publication in [journal] and is subject to Institution of Engineering and Technology Copyright. The copy of record is available at IET Digital Library 
surface wave null at $2.4 \mathrm{GHz}$, to meet our proposed design goals. To generate the dimensions for grids embedded in a dielectric a scaling factor of $1 / \sqrt{ } \varepsilon_{\mathrm{r}}$ was used. This assumes the grids are embedded in an infinite dielectric but this approximation is valid as there is a small covering layer of dielectric [4]. The values of permittivity used along with the dimensions for each substrate are included in Table 2 . The simulated transmission characteristics, ignoring dielectric losses, are compared in Fig. 6.

Table 2 Details of different dielectric used in study 4

\begin{tabular}{lllllll}
\hline Material & $\boldsymbol{\varepsilon}_{\mathbf{r}}$ & $\tan \delta$ & $\mathrm{P}$ & $\mathrm{W}$ & $\mathrm{S}$ & $\mathrm{T}$ \\
$\mathrm{mm}$ & & $\mathrm{mm}$ & $\mathrm{mm}$ & $\mathrm{mm}$ \\
\hline Air & 1.0 & 0.0 & 139 & 19.0 & 106 & $(20)$ \\
Dry Wall & 2.1 & - & 96.3 & 13.1 & 73.3 & 13.8 \\
Plywood & 2.4 & 0.03 & 90.6 & 12.4 & 68.9 & 13.0 \\
Hollow Concrete & 3.5 & 0.30 & 74.9 & 10.2 & 57.0 & 10.8 \\
Brick & 5.1 & 0.04 & 61.7 & 8.40 & 47.0 & 8.90 \\
Solid Concrete & 7.7 & 0.19 & 50.1 & 6.80 & 38.1 & 7.20 \\
\hline
\end{tabular}

It is seen from Fig. 6 that embedding the gratings in a dielectric causes a narrowing of the lower passband as the permittivity increases. Increasing permittivity also introduces additional 'interactions' around $1800 \mathrm{MHz}$ giving ripple in the response. Indeed there is a small dip at $1800 \mathrm{MHz}$ for $\varepsilon_{\mathrm{r}}=7.7$. Overall the transmission loss is generally below $5 \mathrm{~dB}$ for both passbands, which may be considered low loss for a wave penetration. The surface wave null remains at $2.4 \mathrm{GHz}$ and does show a small widening with increasing permittivity although perhaps remains too narrow to be useful (-20 dB transmission loss bandwidth $\sim 10 \mathrm{MHz}$ - Wifi typically channels are $22 \mathrm{MHz}$ wide from 2.41 to $2.49 \mathrm{GHz}$ ). (note , there are some additional extremely narrow resonances but it is thought these are numerical errors from CST).

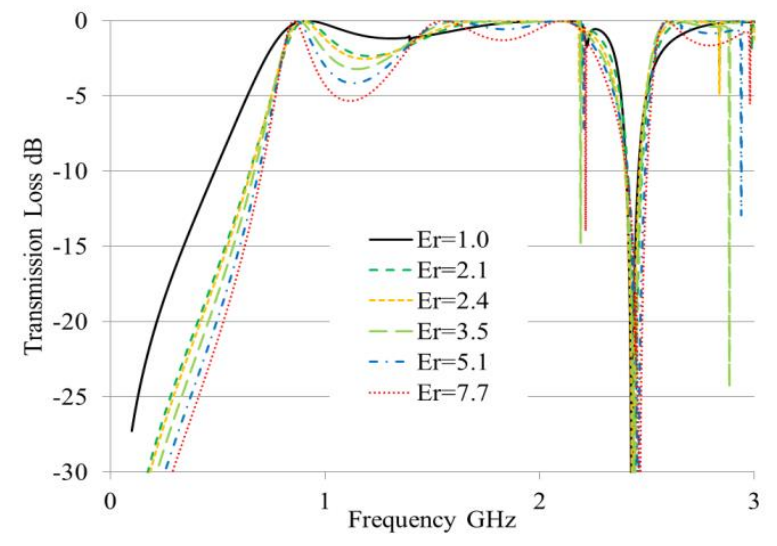

Fig.6: Effect of Dielectric on Grid Filter Response (Normal Incidence)

This paper is a postprint of a paper submitted to and accepted for publication in [journal] and is subject to Institution of Engineering and Technology Copyright. The copy of record is available at IET Digital Library 
To a first order scaling the dimensions accommodates the effect of a supporting dielectric, although there is evidence of additional 'interactions' caused by the effect of a finite dielectric thickness which may offer a further degree of freedom in tuning a structure to give a required plane wave transmission response. However, the responses in Fig. 6 are for loss less substrates. In practice the loss tangent for typical building materials can be significant, ranging from 0.03 for brick to 0.19 for concrete. It is also worth noting from [13] $\tan \delta$ for concrete is dependent on the water content - with tan $\delta$ reducing significantly as the concrete dries out. (Note in [2] tan $\delta$ for concrete was taken to be 0.1 ). To investigate the effect of tan $\delta$ simulations were made for the 'concrete' structure $\left(\varepsilon_{\mathrm{r}}=7.7\right)$ but using different values of tan $\delta$. Figure 7 shows that the increase of transmission loss is marginal for $\tan \delta=0.03$ (i.e. for materials such as bricks) but can be significant for concrete, with up to $8 \mathrm{~dB}$ of additional loss in a transmission band.

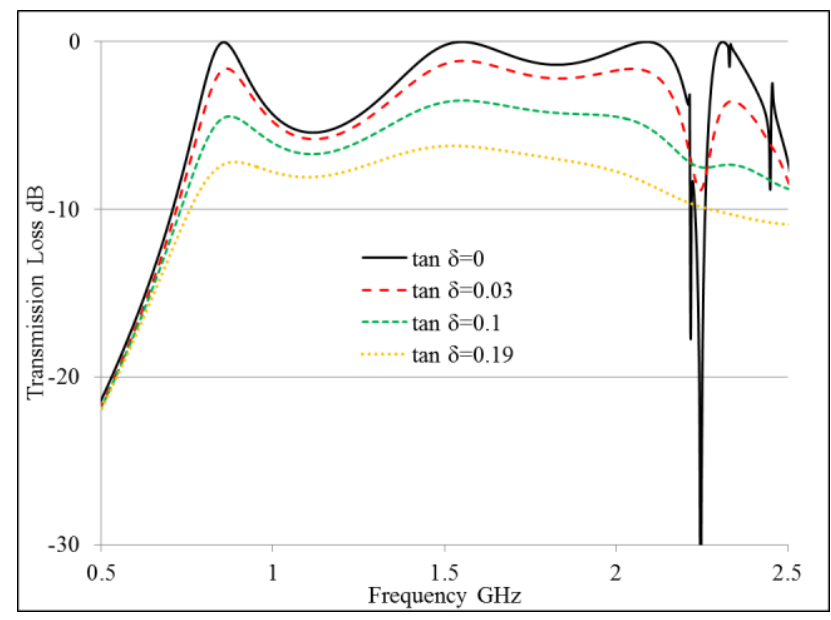

Fig. 7: Effect of Loss Tangent (Normal Incidence)

\section{Effect of Incidence Angle}

The response so far has considered only normal incidence but in practice the waves may enter a room over a range of angles of incidence and so transmission responses were simulated firstly for the airspaced structure in Table 2 at various TE angles of incidence. These are presented in Fig. 8. As might be expected the response changes markedly with incidence angle. The primary effect of the lower passband is seen to increase in frequency and the transmission response deteriorates considerably for incidence angles above $40 \mathrm{o}$, which is not particularly high.

Importantly the $2.4 \mathrm{GHz}$ surface wave resonance exhibits significant changes. Firstly the frequency of the null reduces with incidence angle (as might be expected from [4]). There also appears to be multiple resonances forming above the first surface wave null. Ultimately the surface wave null has a detrimental affect overall. The simple air-spaced structure therefore has limited application.

This paper is a postprint of a paper submitted to and accepted for publication in [journal] and is subject to Institution of Engineering and Technology Copyright. The copy of record is available at IET Digital Library 


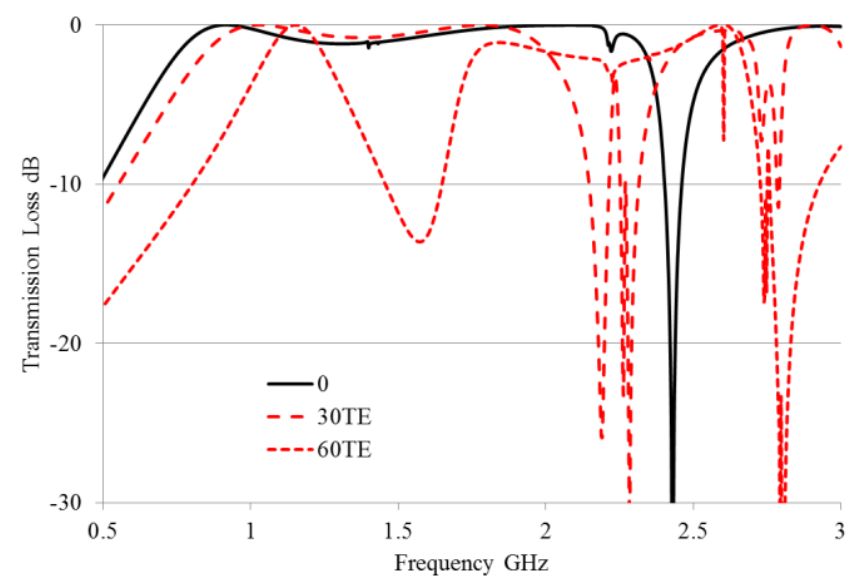

Fig. 8: Transmission Response at different incidence angles for air-spaced double grid filter

It is well known that a dielectric can reduce the effects of incidence angle of an FSS structure [4] and the intention for this structure is that it should be embedded in a wall structure - indeed may even constitute 'reinforced concrete'. Further simulations were made for a structure using a dielectric of permittivity 7.7, using the dimensions from Table 2. Simulations were made for TE and TM planes of incidence up to $60^{\circ}$ and also for loss-less and lossy material. The transmission responses are plotted in Fig. 9.

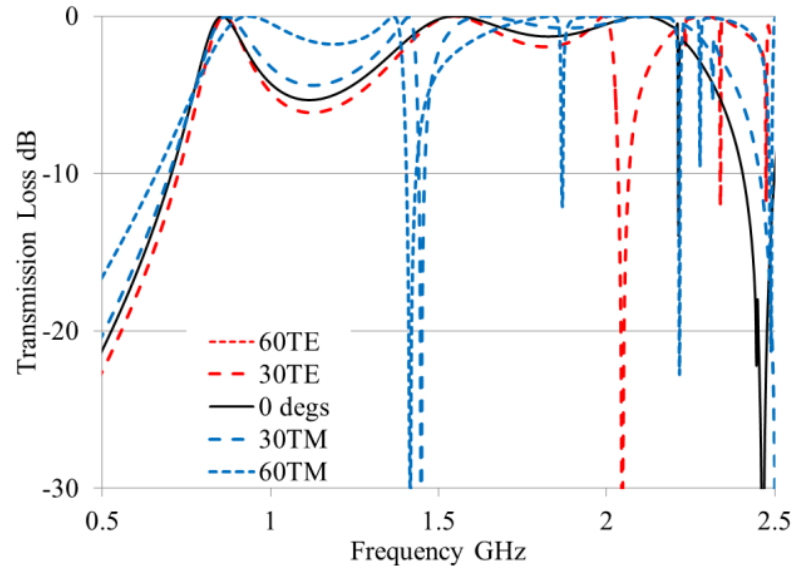

(a)

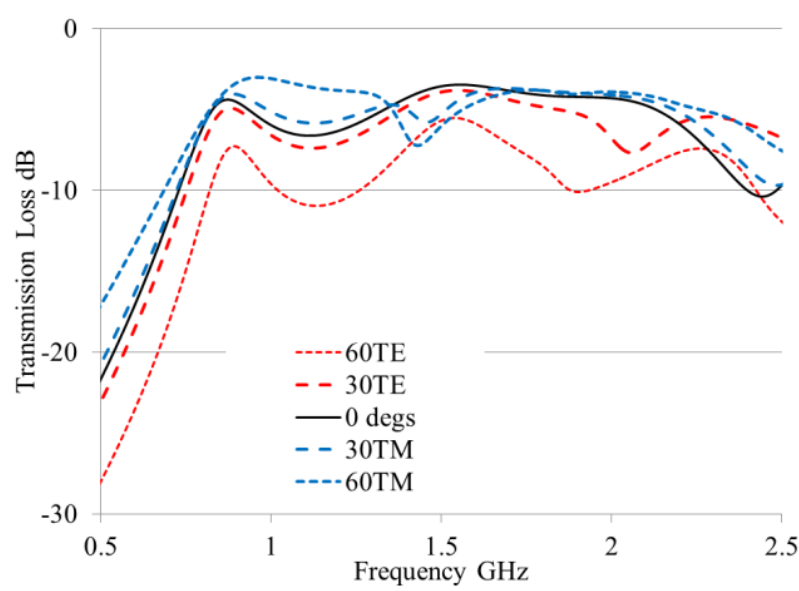

(b))

Fig. 9: Transmission Response for different incidence angles for Double Layer Grid Filter embedded in 'Solid Concrete' $\left(\varepsilon_{r}=7.7\right)$

(a) Loss-less (tan $\delta=0.0)$

(b) Lossy $(\tan \delta=0.1$

From Fig. 9(a) It can be seen that the effect of incidence angle on the resonant frequency of the first transmission band is reduced considerably. The bandwidth of this transmission band also reduces with increasing angle in the TE plane whilst widening in the TM plane, again typical of a single layer FSS embedded in dielectric [4]. The response at higher frequencies (around $1.8 \mathrm{GHz}$ ) is again reasonably stable

This paper is a postprint of a paper submitted to and accepted for publication in [journal] and is subject to Institution of Engineering and Technology Copyright. The copy of record is available at IET Digital Library 
but again suffers from the appearance of surface wave nulls at much lower frequencies. Interestingly in the TE plane the nulls are at a higher frequency, but for TM nulls appear as low as $1.5 \mathrm{GHz}$

The response of Fig. 9(a) is to be compared to Fig. 9(b) which shows the additional effect of dielectric losses as incidence angle is varied. Here a value of 0.1 was used for tan $\delta$ as a 'good' value for concrete - in practice it may be higher than this. As seen before in Fig. 7 there is a significant increase in loss due to absorption, with typically $5 \mathrm{~dB}$ transmission loss rising to $10 \mathrm{~dB}$ at 60TE incidence. The increase of loss is related to a reduction in the resonance bandwidths as noted for single layer FSS [14]. Of interest the surface wave nulls are no longer apparent.

\section{Reduced Periodicity}

Whilst one of the design goals was to use a surface wave null to provide a reflection band it is clear this effect is very sensitive to incidence angle such that it can cause losses in a waned passband. Naturally it should be possible to use a grid with a smaller periodicity, $\mathrm{P}$, to avoid any surface waves in the band of interest, and tune the structure for the desired transmission bands. To avoid any surface wave nulls for any angle of incidence the periodicity should be $<\lambda \varepsilon / 2$, where $\lambda \varepsilon$ is the wavelength at the highest frequency inside the dielectric. For our design example with $\varepsilon_{\mathrm{r}}=7.7$, this requires $\mathrm{P}$ to be $22.5 \mathrm{~mm}$. As a first approximation the 'inductance' of a grid is proportional to the ratio $\mathrm{W} / \mathrm{P}$, so for an investigation into a smaller periodicity $\mathrm{W}$ was scaled from $6.80 \mathrm{~mm}$ to $3.05 \mathrm{~mm}$. However, the dielectric spacing was kept at $38.1 \mathrm{~mm}$. Simulations for this modified structure are shown in Fig 10, assuming a loss less dielectric.

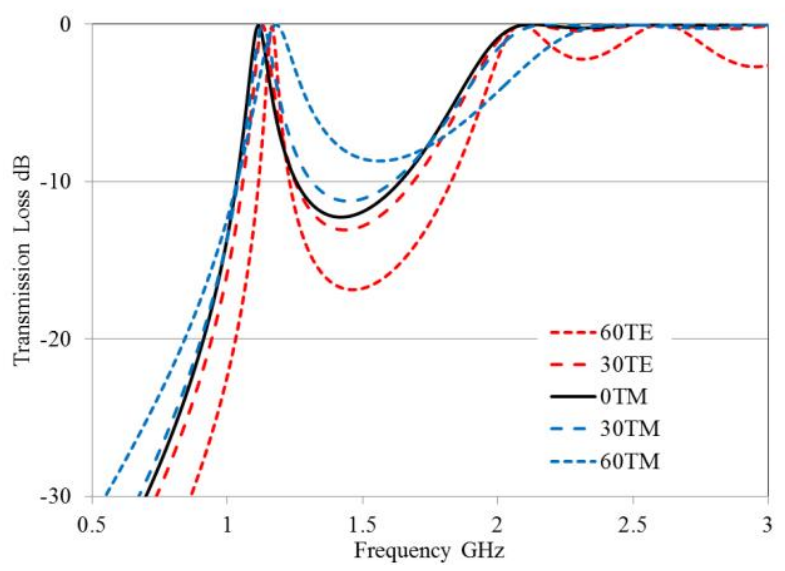

Fig.10: Transmission Response of Reduced Periodicity Structure Embedded in lossless 'Concrete' $\left(\varepsilon_{r}=7.7, P=22.5 m m\right.$, $W=3.05 \mathrm{~mm}, S=38.1 \mathrm{~mm}$ )

The reduced periodicity structure no longer exhibits any surface wave null effects over the band of interest (up to $2.4 \mathrm{GHz}$ ). However $900 \mathrm{MHz}$ and $1800 \mathrm{MHz}$ transmission bands differ from the previous design. Firstly they are at a higher frequency $(1.1 \mathrm{GHz}$ rather than $0.9 \mathrm{GHz})$ and the bandwidth has also

This paper is a postprint of a paper submitted to and accepted for publication in [journal] and is subject to Institution of Engineering and Technology Copyright. The copy of record is available at IET Digital Library 
been reduced. Clearly simply scaling the grid dimensions is only an approximation and underlines the need to use full-wave simulations rather than simple equivalent circuit models. However Fig. 10 does show that it is possible to tune a dielectric 'wall' using 2 grids to provide defined transmission bands. It is seen these are quite stable up to $60^{\circ}$ of incidence angle.

\section{Effect of Tolerance}

A major reason for considering this grid structure is the simplicity for manufacture. Typical single layer FSS solutions require forming intricate patterns that has been difficult to realize in practice. This approach could be created by simply fixing a metallic mesh on either face of a solid wall - even extending over adjacent walls and possibly onto the floors and ceiling. As such there will still be mechanical tolerances to consider. To evaluate this simulations of the 'solid concrete' structure of Table 2 at normal incidence were made but with small variations of the various parameters. OF importance is the stability of the first resonance so the frequency of this was plotted against mechanical error in Fig. 11.

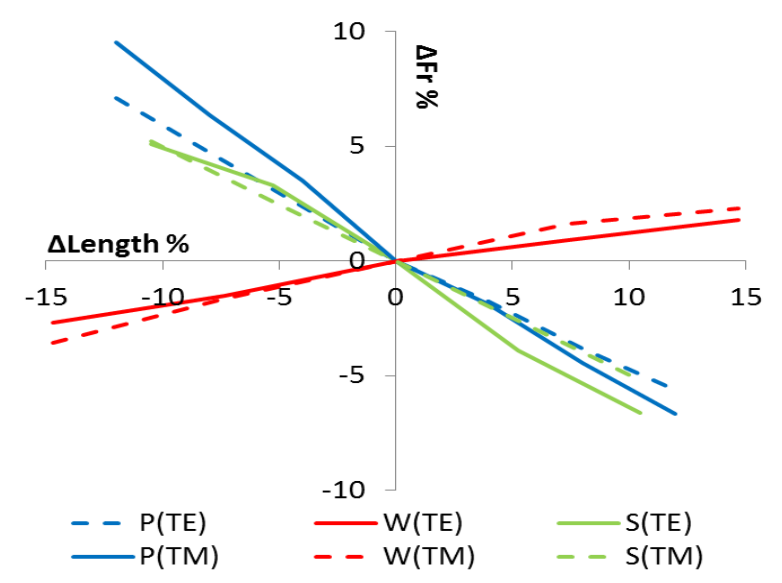

Fig. 11: Effect of Mechanical Tolerances on the Frequency of the First Resonance

From Fig. 11 it is seen that the resonance has less than 5\% shift for $10 \%$ error of the periodicity or the grid spacing and even less with changes in the width of the grid wires. In Practice the width should be controlled to less than $2 \%(0.1 \mathrm{~mm})$ as should the periodicity $(<1 \mathrm{~mm})$. The largest source of error will be from the dielectric separating the grids. If this is a building material it may be difficult to keep the thickness accurate to less than $5 \%$ with the added complication of variability of the dielectric constant (resonance is directly proportional to $\sqrt{ } \varepsilon_{\mathrm{r}}$ ).

This paper is a postprint of a paper submitted to and accepted for publication in [journal] and is subject to Institution of Engineering and Technology Copyright. The copy of record is available at IET Digital Library 


\section{Experimental Double Layer Grid Filter}

To evaluate the proposed structure a prototype was constructed using the initial air-spaced dimensions of $\mathrm{W}=19 \mathrm{~mm}, \mathrm{P}=139 \mathrm{~mm}$ and $\mathrm{S}=106 \mathrm{~mm}$ (i.e. air-spaced structure Table 2). The grids were constructed by fixing $19 \mathrm{~mm}$ wide, $30 \mu \mathrm{m}$ thickness adhesive copper tape onto $4 \mathrm{~mm}$ thickness hardboard panels of overall size $2.4 \mathrm{~m} \times 2.4 \mathrm{~m}$. The two panels were held apart using a wooden frame. The experimental hardware is shown in Fig. 12 placed inside an anechoic chamber midway between wideband Vivaldi antennas spaced $5 \mathrm{~m}$ apart. The Vivaldi antennas had a maximum dimension of $250 \mathrm{~mm}$, with an operating band of $400 \mathrm{MHz}$ to $3 \mathrm{GHz}$, placing the panel in the far field of each antenna. Conversely the antennas were in the 'near field' region of the panel, to respond to a plane wave at the surface. The measured 'plane wave' transmission response for $\sim 50$ is presented in Fig. 13 (solid line) for comparison to the expected response simulated using CST - assuming infinite arrays. (Note measurement is made at 'near-normal incidence' as reflections at normal incidence can create standing waves in the set-up).

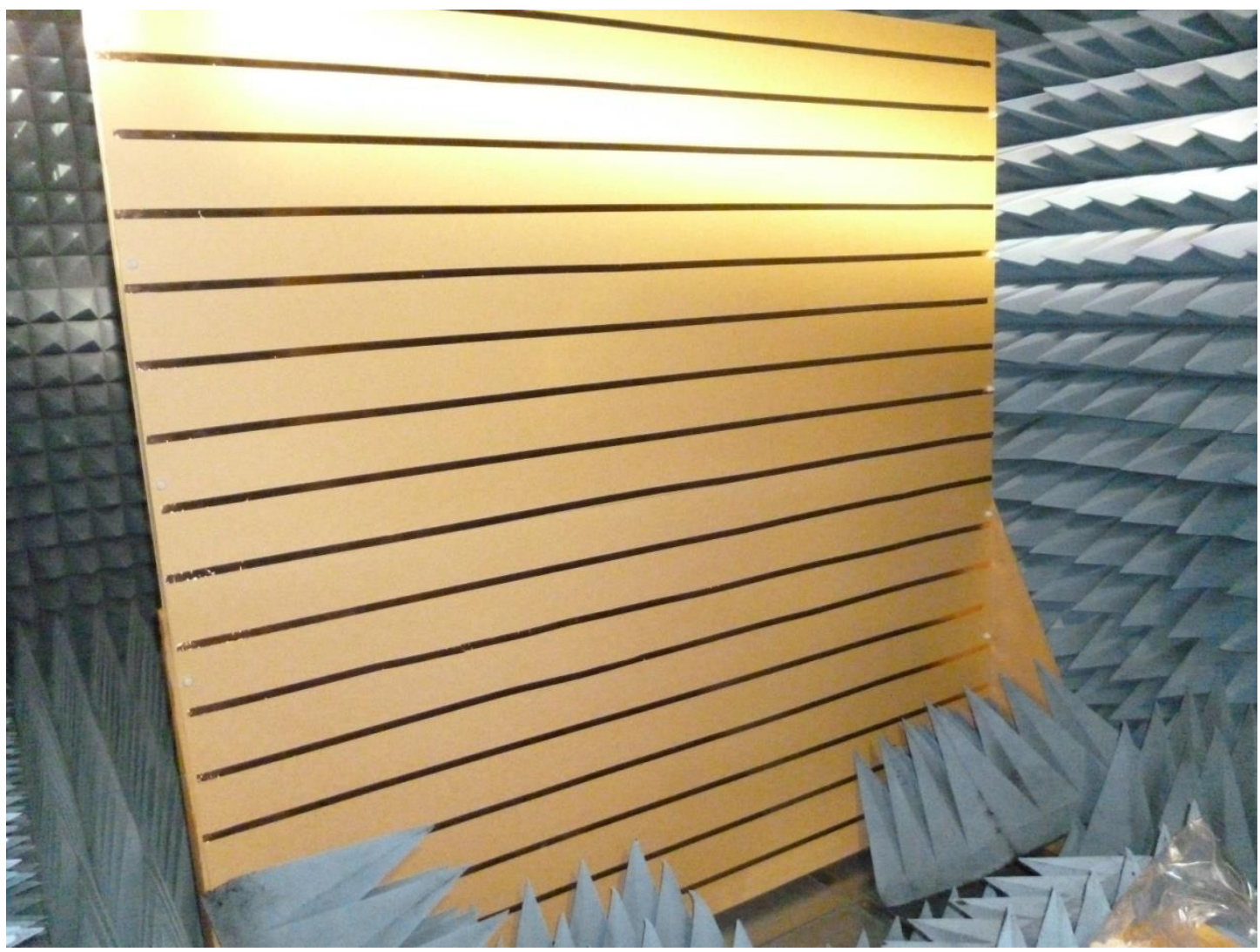

Fig. 12: Photo of Experimental 1m Panel under Test

The measured response shows reasonable agreement to the model, particularly at higher frequencies. For normal incidence the first surface wave was designed to be at $2.4 \mathrm{GHz}$, with a second null appearing at $2.83 \mathrm{GHz}$ due to higher order grating modes. The measured response exhibits nulls at much lower

This paper is a postprint of a paper submitted to and accepted for publication in [journal] and is subject to Institution of Engineering and Technology Copyright. The copy of record is available at IET Digital Library 
frequencies with the first at $2.05 \mathrm{GHz}$, a second at $2.3 \mathrm{GHz}$ and now a third at $2.65 \mathrm{GHz}$. However, it was noted the surface waves nulls are very sensitive to incidence angle for free-standing grids and it is found close agreement is found between simulation and the measurement if an incidence angle of $7^{\circ}$ is used in the model. The nulls are also less well defined (wider bandwidth, not so deep) which is likely due to the finite size of the panels and that they were not perfectly flat. At low frequencies the measurement is less well defined although it displays a dip in transmission around $1.3 \mathrm{GHz}$ and a peak around $900 \mathrm{MHz}$, a designed passband. As the frequency reduces the plane wave approximation is poorer hence the measurement exhibits larger differences. This is caused by the finite size of the grids (less than 10 wavelengths below $1.25 \mathrm{GHz}$ ) and non-uniform illumination of the grids due to the beamwidth of the Vivaldi antennas. Indeed some scattering effects are evident by transmission greater than OdB - due to diffraction off the edge of the finite sized panel.

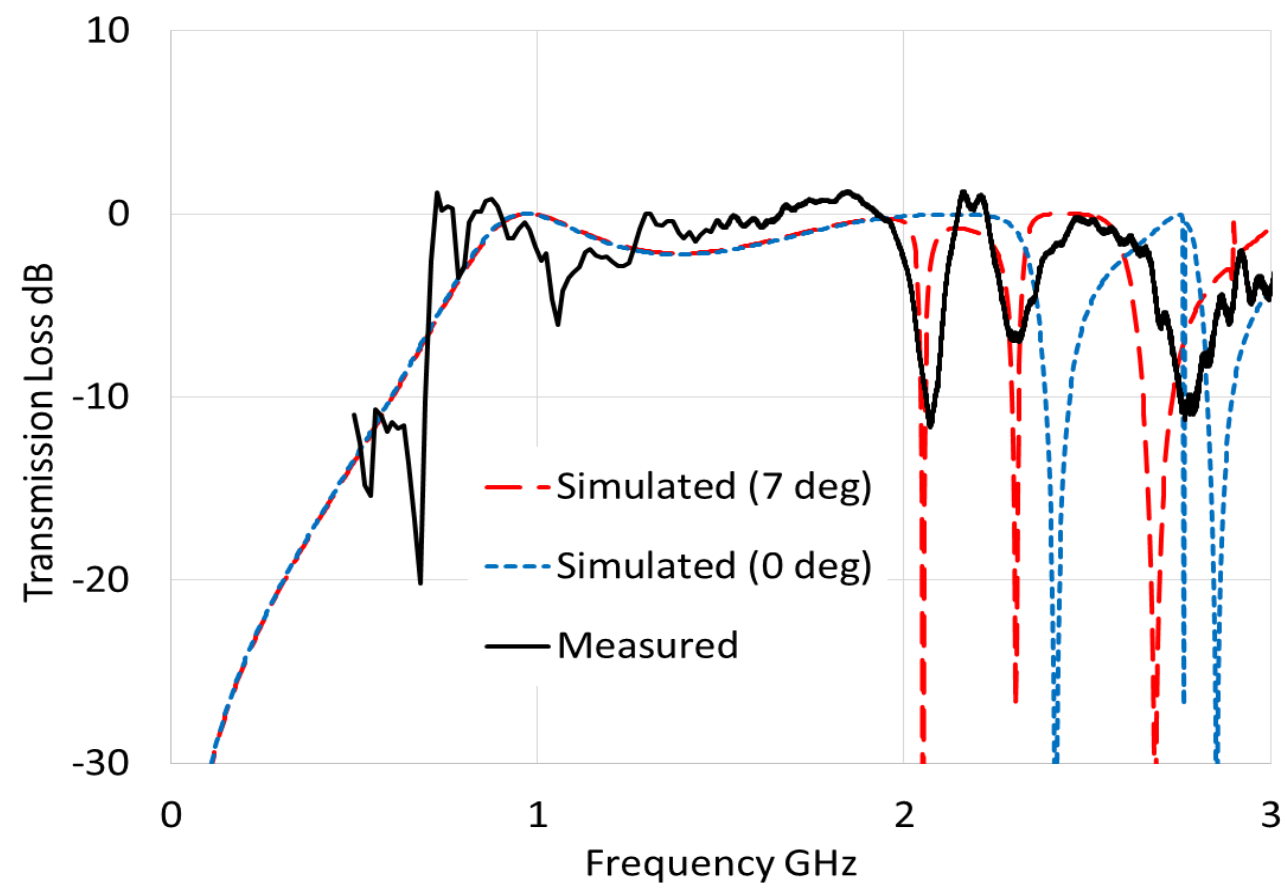

Fig. 13: Plane Wave Transmission Response of 1 m Panel (near-normal incidence)

\section{Discussion}

A goal of this work was to consider if the structure of steel reinforced concrete could be 'tuned' to permit plane wave transmission at specific wireless bands. In principle this has been demonstrated but actual implementation will be limited in practice for several reasons. Typical steel meshes are 6-10 $\mathrm{mm}$ in diameter with a periodicity of $200 \mathrm{~mm}$. This gives a value of W/P of 0.01 compared to 0.14 for the design

This paper is a postprint of a paper submitted to and accepted for publication in [journal] and is subject to Institution of Engineering and Technology Copyright. The copy of record is available at IET Digital Library 
in Table 2, so the metal mesh may give a suitable 'inductive' effect. However, the periodicity of $200 \mathrm{~mm}$ will mean surface waves nulls begin as low as $300 \mathrm{MHz}$ for $\varepsilon_{\mathrm{r}}=7.7$ ! Of course it should be possible to use a smaller sized mesh (10 times smaller!) - but there may be an impact on the structural strength, weight, cost, etc. that needs investigating. For the designs considered here the concrete wall had an overall thickness of $50 \mathrm{~mm}$ - previous work [2] considered thicknesses of $300 \mathrm{~mm}$. The dielectric effect would be significantly greater - but may be compensated by using multiple grids, each separated by approximately $\lambda / 2$. This could be a topic for further research. Lastly a major problem with concrete was the high loss factor, resulting in significant transmission absorptive loss that cannot be 'tuned' out. This factor could limit practical application to tuning reinforced concrete structures.

Whist it may not be possible to use this technique on reinforced concrete to provide transmission bands, the approach could have significant benefits on walls constructed from materials such as brick, having a high permittivity but low loss tangent. For this type of wall the mesh would need to be fixed to the surface. In this instance the thickness of the wall would be fixed but it should still be possible to tune the mesh to provide suitable transmission bands. It is noted from [4] that the effective permittivity would be the 'average' of the adjacent materials, and also the onset of surface wave nulls would depend on the highest permittivity (e.g. the brick).

\section{Conclusion}

It has been shown that introducing a second wire mesh into a wire loaded material can provide transmission bands, offering a solution to the problem of obstruction of wireless signals by reinforced building materials. Here 2 free standing gratings were shown to provide transmission bands at $900 \mathrm{MHz}$ and $1800 \mathrm{MHz}$. Embedding these gratings in a dielectric gave two benefits. Firstly transmission properties of the dielectric itself can add additional transmission bands, offering transmission to most mobile bands. Secondly the presence of a dielectric reduced the effect of incidence angle.

Surface wave nulls are expected with periodic structures but in this work the use of this phenomenon was considered to provide reflection bands to contain WiFi /WLAN signals at $2.45 \mathrm{GHz}$. This was demonstrated in principle, but in practice the surface wave null has too narrow a bandwidth and also was found to be too sensitive to angle of incidence, even in the presence of a dielectric.

The challenge of controlling propagation in buildings fundamentally rests in presenting a solution that can be realized. An easy to construct solution has been presented that may be used to enhance transmission through high permittivity and metal reinforced building materials.

This paper is a postprint of a paper submitted to and accepted for publication in [journal] and is subject to Institution of Engineering and Technology Copyright. The copy of record is available at IET Digital Library 


\section{Acknowledgments}

The authors would like to thank Becky Callaghan for her assistance with the measurements.

\section{References}

[1] A. Newblold: 'Designing buildings for the wireless-age', IEE Communications Engineer , Vol. 2, Iss. 3, pp 1821,2004

[2] M Philippakis, C Martel, D Kemp: 'Application of FSS structures to selectively control the propagation of signals into and out of buildings Annex 4: In-building propagation enhancement' Ofcom ref. AY4464A, 2004 [Online] Available: http://stakeholders.ofcom.org.uk- /binaries/research/spectrum-research/in_building.pdf

[3] J. Batchelor, E.A. Parker, B. Sanz-Izquierdo, J.-B Robertson, and I. Ekpo, "Designing FSS for wireless propagation control within buildings", Antennas \& Propagation Conference, 2009. LAPC 2009. Loughborough, pp $14-17$

[4] Callaghan, P., Parker, E.A. \& Langley, R.J.: 'Influence of supporting dielectric layers on the transmission properties of frequency selective surfaces', IEE Proc., Pt. H, Vol.138, No.5, Oct 1991, pp448-454

[5] B. Sanz-Izquierdo, E. A. Parker, J.-B. Robertson, and J. C. Batchelor, "Singly and dual polarized convoluted frequency selective structures,” IEEE Trans. Antennas Propag., vol. 58, no. 3, pp. 690-696, Mar. 2010

[6] F. Deng ; X. Yi \& W. Wu: 'Design and Performance of a Double-Layer Miniaturized-Element Frequency Selective Surface', IEEE Antennas and Wireless Propagation Letters, Vol. 12, 2013 , pp 721-724

[7] S.I. Sohail, K.P. Esselle, G Kiani, "Design of a bandpass FSS on dual layer energy saving glass for improved RF communication in modern buildings", IEEE Antennas and Propagation Society International Symposium (APSURSI), 2012, pp1-2

[8] I-G Leeo, I-P. Hong: 'Frequency Selective Surface Design for Blinds Applications', 7th European Conference on Antennas and Propagation, 2013, pp 2753-2755

[9] N. Qasem \& Rob Seager; 'Overcoming the influence of people shadowing and enhancing MIMO capacity systems via modified environments', Antennas \& Propagation Conference, 2012. LAPC 2012. Loughborough, pp 14

[10] C. Christodoulou, D. P. Kwon, R. Middleveen and P.F. Wahid: 'Scattering from stacked gratings and dielectrics for various angles of wave incidence', IEEE Trans Vol. TAP 36, No. 10 October 1988 pp 1435-1441

[11] A.W. Rudge, K. Milne, A.D. Olver, P. Knight: 'The handbook of Antenna Design', Published Peter Peregrinus, 1986, ISBN 0864310529, Ch. 14, pp1156-1157

[12] N Marcuvitz: 'Waveguide Handbook', Published Peter Peregrinus, 1993, ISBN 0863410588, Ch 5, pp284-285

[13] C. Thajudeen, A. Hoorfar, and F. Ahmad: 'Measured complex permittivity of walls with different hydration levels and the effect on power estimation of TWRI target returns', Progress In Electromagnetics Research B, Vol. 30, 2011, pp177-199

This paper is a postprint of a paper submitted to and accepted for publication in [journal] and is subject to Institution of Engineering and Technology Copyright. The copy of record is available at IET Digital Library 
[14] Callaghan, P. \& Parker, E.A.: 'Loss-Bandwidth Product for Frequency Selective Surfaces', Electronic Letters, 1992, 28, pp. 365

This paper is a postprint of a paper submitted to and accepted for publication in [journal] and is subject to Institution of Engineering and Technology Copyright. The copy of record is available at IET Digital Library 